\title{
Paediatric Asthma Management in the Kingdom of Saudi Arabia: The need for Culturally Sensitive Research and Practice
}

\author{
Abeer Alatawi ${ }^{1 *}$, Pam Smith ${ }^{2}$, Nicola Ring ${ }^{3}$ and ColinChandler ${ }^{4}$ \\ ${ }^{1}$ Department of Nursing, Tabuk University, United Kingdom \\ ${ }^{2}$ Department of Nursing Studies, The University of Edinburgh, United Kingdom \\ ${ }^{3}$ Child Health Nursing, Edinburgh Napier University, United Kingdom \\ ${ }^{4}$ Department of Nursing Studies, The University of Edinburgh, United Kingdom
}

*Corresponding author: : Abeer Alatawi, Assistant Professor- Child Health Nursing Department of Nursing, Faculty of Applied Medical Sciences, Tabuk University, United Kingdom.

To Cite This Article: Abeer Alatawi.Paediatric Asthma Management in the Kingdom of Saudi Arabia: The need for Culturally Sensitive Research and Practice. Am J Biomed Sci \& Res. 2019 - 6(2). AJBSR.MS.ID.001006. DOI: 10.34297/AJBSR.2019.06.001006.

Received: 眥 November 6, 2019; Published: 眥 November 12, 2019

\begin{abstract}
Asthma is the third most common chronic disease in Saudi Arabia, affecting two million people. Asthma is also a leading cause of death among allergic disorders. Uncontrolled asthma may considerably decrease the quality of life for children and their families. Over the years, the Saudi government has included asthma as a major concern in their strategic health plan and has encouraged research in this area. However, paediatric asthma management remains under-researched and needs to be investigated further in the Saudi context. This review summarizes the recent advances in the paediatric asthma management in Saudi Arabia, including the need for culturally sensitive research and practice. The authors discuss the recent context of asthma management in KSA. The authors discuss the need for culturally and ethnically sensitive asthma management research and practice.
\end{abstract}

Keywords: Asthma; Management; Children; Saudi Arabia; Culturally sensitive care

\section{Introduction}

Asthma is a potentially life-threatening condition and is one of the most common respiratory problems causing lung airway obstruction [1]. It is a disease which has no cure but can be controlled by use of proper medication and effective selfmanagement through education [2]. In 2010 an estimated 300 million people were currently asthmatic globally [3]. Poor control of asthma may also lead to frequent use of emergency departments, hospitalisation, and, in severe cases, death. Despite several decades of advances in its control and management, asthma remains a common worldwide health and socio-economic problem [4].

Approximately 1.4 million children aged $2-15$ in the UK are currently receiving treatment for asthma [5]. In the US, nine million children aged under 18 years have asthma [6]. Asthma is an increasingly significant child health problem within the Kingdom of Saudi Arabia (KSA). Although the prevalence rate of asthma among Saudi adults is unknown, estimations by the Saudi Initiative for Asthma (SINA) [7] placed the overall prevalence rate among Saudi children between 8-25 \% in 2012 [8]. The prevalence of lifetime wheeze, wheeze over one year and physician-diagnosed asthma in children have been reported as $25.3 \%, 18.5 \%$ and 19.6\%, respectively [9]. According to international statistics, in 2016 it is estimated that the Saudi population will comprise $29.4 \%$ citizens aged under 15 , and $67.6 \%$ aged $15-64$. This indicates that paediatric asthma continues to be a major public health concern in KSA, especially as asthma control for many patients remains suboptimal. For example, a recent asthma control survey conducted in KSA showed that only $5 \%$ of child and adult patients were controlled, $31 \%$ were partially controlled, and $64 \%$ were uncontrolled [10].

Another recent cross-sectional study conducted among children with asthma aged 4-11 years revealed that uncontrolled asthma was present in about $59.3 \%$ of children [11]. This situation has considerable consequences for children in reducing their quality of 
life through increased hospitalisations and emergency visits [12]. For children, uncontrolled and poorly managed asthma also results in frequent school absences, affecting their educational attainment and future employment prospects and general life outcomes, including health [13].

\section{The context of asthma management in KSA}

Internationally, achieving effective asthma management can be challenging for health professionals for a range of reasons, including under-diagnosis, lack of patient education, poor health facilities and limited treatment choices [14-15]. Such issues make asthma management even more challenging in KSA. For example, although there are available written protocols for the diagnosis and management of asthma in the country, there is still inadequate knowledge and application of these guidelines among health care providers [16,17]. Overall, the level of awareness of general knowledge, diagnosis, classification of severity, and management among physicians is considered average (52\%), while that of other health professionals in general is considered low [7].

A shortage of Saudi healthcare professionals means that KSA has long employed doctors and nurses from other countries, resulting in the majority of healthcare professionals in KSA being expatriates. There are also issues in KSA which make the professional management of asthma more challenging. The Saudi Ministry of Health $(\mathrm{MoH})$ for example reported that only $38 \%$ of its total health workforce were Saudis [18]. The use of health professionals from other countries contributes to high employee turnover rates and can cause barriers during clinical consultations, as foreign professionals usually lack Arabic language skills to communicate with children and parents $[19,20]$.

In many other countries, including the UK and USA, asthma management services are provided by doctors and nurses working collaboratively. Nurses have an active role in the long-term asthma management and education of patients [21], but this is not the case in KSA where nursing staff typically have a negligible, ad hoc role in asthma management [8]. As the role of nurses in asthma care in KSA is under-developed, the burden of asthma management in Saudi therefore primarily falls on doctors rather than being shared with nurses. In recognition of this medical load, and the need to develop asthma care, SINA asthma guidelines were introduced in 2009 to mirror other national and international guidelines like GINA and NAEPP $[8,22,23]$, particularly highlighting the importance of good asthma education [7].

One of the specific challenges of providing effective paediatric asthma management in the KSA is inadequate awareness of the condition. An estimated $50 \%$ of the populations of the major Saudi cities of Riyadh Jeddah and Dammam do not know asthma symptoms [24]. Public awareness campaigns regarding asthma, its symptoms, medications, and consequences are urgently required in KSA with specific initiatives targeted towards parents of children with asthma, because parental lack of asthma knowledge is likely to contribute to delays in children accessing health services and receiving asthma treatment which may lead to higher morbidity and mortality rates [13]. To that end, an on-going, nationally funded nurse-led research is being conducted to enable nurses working in KSA to better support doctors in the management of asthma by becoming more involved in the provision of paediatric asthma education, which requires more information about the current nursing role in this field and how it can be improved.

In addition to these contextual barriers to effective paediatric asthma management in KSA, there is also a lack of asthma research specifically conducted in the country. Saudi studies have tended to investigate asthma control or drug use using quantitative methods such as surveys, but rarely cohort designs [12,25-26]. Most Saudi asthma research has focused on the prevalence of asthma among children $[4,9,27,28]$, investigating relationships between asthma and other factors including triggers and symptoms [29-31] and assessment of risk factors [27,32-34]. For instance, there has been a lack of research evaluating asthma management interventions, including the delivery of asthma education programmes for children and parents and their outcomes within a Saudi context. It can be argued that some of the risk factors could be avoided by providing children with sufficient education about asthma. Therefore, there is a need in KSA to educate children with asthma about their illness and to test the effectiveness of these educational programmes in the Saudi context.

\section{The need for culturally and ethnically sensitive asthma management research \& practice}

In recent years recognition has been growing of the need to provide asthma care that is sensitive to the cultural and ethnic needs of patients and parents [35]. A systematic review on barriers and facilitators to asthma management in the care of South Asian children identified some key ethnic and cultural issues, including the impact of parental and professional knowledge and beliefs, health service utilisation pattern explanations and the impact of prejudice and stigmatisation on the improving of asthma management, and language barriers [36]. In the UK, substantial ethnic variations in hospital admission and deaths from asthma have been noted in Scotland [37]. Cultural factors, including self-management and health seeking behaviour as well as variations in the quality of primary care provision, are the most likely explanations for these differences. Such leading research on culturally sensitive care focuses on countries like the UK and USA, where Arabic patients/ parents are an ethnic minority. However, in countries like KSA it is necessary to conduct quantitative research on asthma outcomes (e.g. to determine whether non-Saudi health professionals communicating in English to Arabic children/parents have the same outcomes such as hospital admission rates as Saudi professionals who speak Arabic). While the vast majority of the indigenous population are Arabic nationals, asthma services and management 
are often provided by health professionals recruited from other countries. Even where foreign personnel are Arabic native speakers (e.g. Egyptians and Palestinians), these professionals may have differing beliefs and values to their Arabian patients and may also lack knowledge about Saudi culture.

Culturally and ethnically sensitive asthma care is also needed for general care purposes, so professionals do not misinterpret extraordinary behaviour as Saudi social norms [38]. Asthma management advice and recommendations offered by some professionals may also not fully reflect Islamic beliefs and values, decreasing compliance among clients. When health professionals and patients belong to different cultures, culturebased misunderstandings can influence their relationships and interactions adversely [39]. The structure of society and families may be another factor preventing culturally specific paediatric asthma care in KSA. In Saudi Arabia there are no laws in place defining a minimum age of children, although numerous drafts for possible laws and regulations have been created since 2011 [40]. For instance, children under 12 years old are seen in children's hospitals, whereas children above 12 years are treated in adult hospitals.

The Royal College of Paediatrics and Child Health (RCPCH) in the UK advocates that young people need special support to manage their long-term condition, with particular emphasis on developing positive health behaviours that continue into adulthood [41]. This is in line with the UN Convention on the Rights of the Child, which formally and explicitly acknowledges the rights for children under international law to be recognised as subjects of rights, rather than merely recipients of adult protection, and that their views are entitled to be heard. These values are recognised in international asthma guidelines which, for example, encourage children to be involved in decisions made about their care, where appropriate research on self-management conducted in western countries may not be transferable to a Saudi context as self-management by children may not be possible within cultural conventions (i.e. they are socio-culturally expected and conditioned to adhere to what their parents say).

There are other barriers which may prevent the delivery of culturally sensitive paediatric asthma management in KSA, such as asthma management advice being provided by female/male health professionals to all genders. However, the current literature on culturally and ethnically sensitive asthma care is gender blind or gender neutral, and has totally over looked gender as a factor that could affect asthma care and outcomes in cultural context where the mixing of men and women is generally avoided, such as conservative Islamic and orthodox Jewish communities [42]. In Saudi Arabia, male health professionals never attempt to interview or examine female patients without a chaperone (typically a father, brother or adult son) being present. It is highly recommended to have female nurses present if female parents and child patients are to be accorded full recognition in decision making for collaborative asthma care recommended internationally [38]. Although SINA guidelines make reference to partnerships between patients and healthcare workers, these values are not in-line with the Saudi culture where women and children are still not given full recognition, and as such they are likely not to be involved in asthma management decisions [7].

However, this issue has been over-looked and un-recognised to-date. Despite the fact that SINA guidelines indicated that asthma education program should be conducted by well-trained health care providers with good communication skills to the families, the cultural barriers to this are not acknowledged (e.g. the need for specific education sessions for mothers and for fathers). There is an urgent need for future research into this area to better understand the issue and to identify in what ways asthma management and education can be better tailored to the cultural needs of children and their parents in countries like KSA. Studies of paediatric asthma management also show indications of inadequate culturally sensitive management different settings in Saudi Arabia.

Culturally sensitive asthma care in KSA confers a key role on qualitative research, for instance observation of asthma consultations might enable exploration of whether non-Saudi health professionals are able to offer culturally sensitive asthma care, and how the situation can be improved. During doing a qualitative research, it can be argued that interviews could be harder when women are covered and veiled since it is agreed that studying verbal and non-verbal communications could not be fully understood without obtaining the views and feelings of the people concerned. Obviously, any such disadvantage was greatly reduced for me due to my cultural competence with veiled Saudi women (see section 4.3.2). Nevertheless, I did not merely rely on the participants' memories, but I also distinguished between participants' descriptions in words and their actions to determine any inconsistencies between what they said and what they did.

Asthma knowledge can only be applied effectively with culturally sensitive practices and attitudes adopted by nurses in their interactions with clients [38]. To navigate these cultural issues, interviews with parents of children with asthma and their health professionals enrich the nursing understanding of asthma management and patient education in KSA, providing culturally appropriate, tailored care and avoiding the mismanagement common in developing countries whereby Western (cultural) models are imported and applied wholesale, without consideration of the local context.

\section{Conclusion}

The prevalence of asthma amongst children in KSA is high in comparison to the rest of the world and it is increasing. This results in significant costs for individual children, their families and the country. This paper highlights specific contextual issues which 
make effective paediatric asthma management in KSA challenging. The need for culturally sensitive asthma care has been raised in countries such as the UK, where Arabic patients are a minority. This paper highlights that there is also a need for culturally sensitive asthma care in countries like KSA where Arabic patients are cared for by health professionals recruited from other countries who do not share their culture, beliefs and values. Culturally and ethnically sensitive asthma care is an un-recognised issue within paediatric asthma care generally and needs to be better understood to be fully addressed by qualitative research.

\section{References}

1. Global Initiative for Asthma [GINA] (2019) Global initiative for asthma.

2. National Heart Lung and Blood Institute [NHLBI] (1997) Asthma Expert Panel Report 2. Bethesda: National Institutes of Health.

3. Douglas J (2010) Occupational asthma. Practice Nurse 40(4): 19-20.

4. Nahhas M, Bhopal R, Anandan C, Elton R, Sheikh A (2012) Prevalence of allergic disorders among primary school-aged children in Madinah, Saudi Arabia: two-stage cross-sectional survey. Plos One 7(5): e36848.

5. Asthma UK (2016) Asthma facts and FAQs.

6. Centre for Disease Control and Prevention, National Center for Health Statistics (2008) New estimates for asthma tracked.

7. Saudi Initiative for Asthma [SINA] (2019) The Saudi Initiative for Asthma - 2016 update: Guidelines for the diagnosis and management of asthma in adults and children.

8. Al Moamary MS, Alhaider SA, Idrees MM, Al Ghobain MO, Zeitouni MO, et al. (2016) The Saudi Initiative for Asthma 2012 update: guidelines for the diagnosis and management of asthma in adults and children. Annals of Thoracic Medicine 11(1): 3-42.

9. Al Ghobain MO, Al Hajjaj MS, Al Moamary MS (2012) Asthma prevalence among 16 to 18 year-old adolescents in Saudi Arabia using the ISAAC questionnaire. BMC Public Health 12: 239.

10. Al Jahdali HH, Al Hajjaj MS, Alanezi MO, Zeitoni MO, Al Tasan TH (2008) Asthma control assessment using asthma control test among patients attending 5 tertiary care hospitals in Saudi Arabia. Saudi Medical Journal 29(5): 714-717.

11. BinSaeed AA, Torchyan AA, Alsadhan AA, Almidani GM, Alsubaie AA, et al. (2014) Determinants of asthma control among children in Saudi Arabia. Journal of Asthma 51(4): 435-439.

12. Asthma and Allergy Foundation of America (2018) Asthma: facts \& figures.

13. Alreshidi NM (2015) The impact of a school-based, nurse-delivered asthma health education programme on quality of life, knowledge and attitudes of Saudi children with asthma. PhD thesis, University of Salford.

14. Leickly FE, Wade SL, Crain E, Kruszon Moran D, Wright EC, et al. (1998) Self-reported adherence, management behavior, and barriers to care after an emergency department visit by inner city children with asthma. Pediatrics 101(5): E8.

15. Speight AN, Lee DA, Hey EN (1983) Speight A N, Lee D A, Hey E N Underdiagnosis and undertreatment of asthma in childhood. British Medical Journal 286(6373): 1253-1256.

16. Coates JR, Steven ID, Beilby J, Coffey G, Litt JC, et al. (1994) Knowledge of and reported asthma management among South Australian general practitioners. British Journal of General Practice 44(380): 123-126.

17. Ahmed AE, Al Jahdali H, Al Harbi A, Khan M, Ali Y, et al. (2014) Factors associated with poor asthma control among asthmatic patient visiting emergency department. The Clinical Respiratory Journal 8(4): 431-436.
18. Saudi Ministry of Health (2010) Health statistics annual. (Accessed on 13 Oct 2015)

19. Aldossary A, While A, Barriball L (2008) Health care and nursing in Saudi Arabia. International Nursing Review 55(1): 125-128.

20. Simpson JL, Scott R, Boyle MJ, Gibson PG (2006) Inflammatory subtypes in asthma: Assessment and identification using induced sputum. Respirology 11(1): 54-61.

21. Zemek R, Plint A, Osmond MH, Kovesi T, Correll R, et al. (2012) Triage nurse initiation of corticosteroids in pediatric asthma is associated with improved emergency department efficiency. Pediatrics 129(4): 671-801.

22. Bousquet J, Clark TJ, Hurd S, Khaltaev N, Lenfant C, et al. (2007) GINA guidelines on asthma and beyond. Allergy 62(2): 102-112.

23. Bateman ED, Boushey HA, Bousquet J, Busse WW, Clark TJ, et al. (2004) Can guideline-defined asthma control be achieved? The Gaining Optimal Asthma Control study. American journal of respiratory and critical care medicine 170(8): 836-844.

24. Al Harbi S, Al Harbi AS, Al Khorayyef A, Al Qwaiee M, Al Shamarani A, et al. (2016) Awareness regarding childhood asthma in Saudi Arabia. Annals of Thoracic Medicine 11(1):60-65.

25. Al Anazi A, Al Moamary M, Ismaeli T, Alanazi TN, Olayan LH, et al. (2015) Asthma in the pediatric population: level of perception among the parents and guardians. International Journal of Medicine and Public Health 5(1): 14-18.

26. Anandan C, Nurmatov U, Van Schayck OCP, Sheikh A (2010) Is the prevalence of asthma declining? Systematic review of epidemiological studies. Allergy 65(2): 152-167.

27. Al Ghamdy YS, Al Haddad NS, Abdelgadir MH, Qureshi NA, Saleh MA, et al. (2000) Socioclinical profile of children with asthma in Al-Majmaah Health Province. Saudi Medical Journal 21(9): 847-851.

28. Al Frayh A, Shakoor Z, Elrab M, Hasnain S (2001) Increased prevalence of asthma in Saudi Arabia. Annals of Allergy, Asthma and Immunology 86(3): 292-296

29. Bazzi M, Sultan M, Al Tassan N, Alanazi M, Al Amri A, et al. (2011) Interleukin 17A and $\mathrm{F}$ and asthma in Saudi Arabia: gene polymorphisms and protein levels. Journal of Investigational Allergology and Clinical Immunology 21(7): 551-555.

30. Farchi S, Forastiere F, Agabiti N, Corbo G, Pistelli R, et al. (2003) Dietary factors associated with wheezing and allergic rhinitis in children. European Respiratory Journal 22(5): 772-780.

31. Hijazi N, Abalkhail B, Seaton A (2000) Diet and childhood asthma in a society in transition: a study in urban and rural Saudi Arabia. Thorax 55(9): 775-779.

32. Al Dawood K (2001) Parental smoking and the risk of respiratory symptoms among schoolboys in Al-Khobar City, Saudi Arabia. Journal of Asthma 38(2): 149-154.

33. Alshehri MA, Abolfotouh MA, Sadeg A, Najjar Y, Asindi AA, et al (2000). Screening for asthma and associated risk factors among urban schoolboys in Abha City. Saudi Medical Journal 21(11): 1048-1053.

34. Hijazi N, Abalkhail B, Seaton A (1998) Asthma and respiratory symptoms in urban and rural Saudi Arabia. European Respiratory Journal 12(1): 41-44.

35. Davidson E, Liu JJ, Sheikh A (2010) The impact of ethnicity on asthma care. Primary Care Respiratory Journal 19(3): 202-208.

36. Lakhanpaul M, Bird D, Manikam L, Culley L, Perkins G, et al. (2014) A Systematic review of explanatory factors of barriers and facilitators to improving asthma management in South Asian children. BioMed Central Public Health 14: 403.

37. Sheikh A, Steiner MFC, Cezard G, Bansal N, Fischbacher C, et al. (2016) Ethnic variations in asthma hospital admission, readmission and death: 
a retrospective, national cohort study of 4.62 million people in Scotland. BioMed Central Medicine 14: 3.

38. Al Shahri MZ (2002) Culturally sensitive caring for Saudi patients. Journal of Transcultural Nursing 13(2): 133-138.

39. Karout N, Abdelaziz SH, Goda M, AlTuwaijri S, Almostafa N, et al. (2013) Cultural diversity: a qualitative study on Saudi Arabian women's experience and perception of maternal health services. Journal of Nursing Education and Practice 3(11): 172.
40. Human Rights Commission in Saudi Arabia (2013) Saudi Arabia 2013 human rights report.

41. Royal College of Paediatrics and Child Health [RCPCH] (2006) Standards for development of clinical guidelines and implementation in paediatrics and child health. 3rd edition. London: Royal College of Paediatrics and Child Health.

42. Al-Khateeb, SA (2008) Women, family and the discovery of oil in Saudi Arabia. Marriage and Family Review 27(1-2): 167-189. 